\title{
Audio Signal Recognition for Speech, Music, and Environmental Sounds
}

(1) Pattern Recognition for Sounds

(2) Speech Recognition

(3) Other Audio Applications

(4) Observations and Conclusions

\author{
Dan Ellis <dpwe@ee.columbia.edu> \\ Laboratory for Recognition and Organization of Speech and Audio \\ (LabROSA) \\ Columbia University, New York \\ http://labrosa.ee.columbia.edu/




\section{Pattern Recognition for Sounds}

- Pattern recognition is abstraction

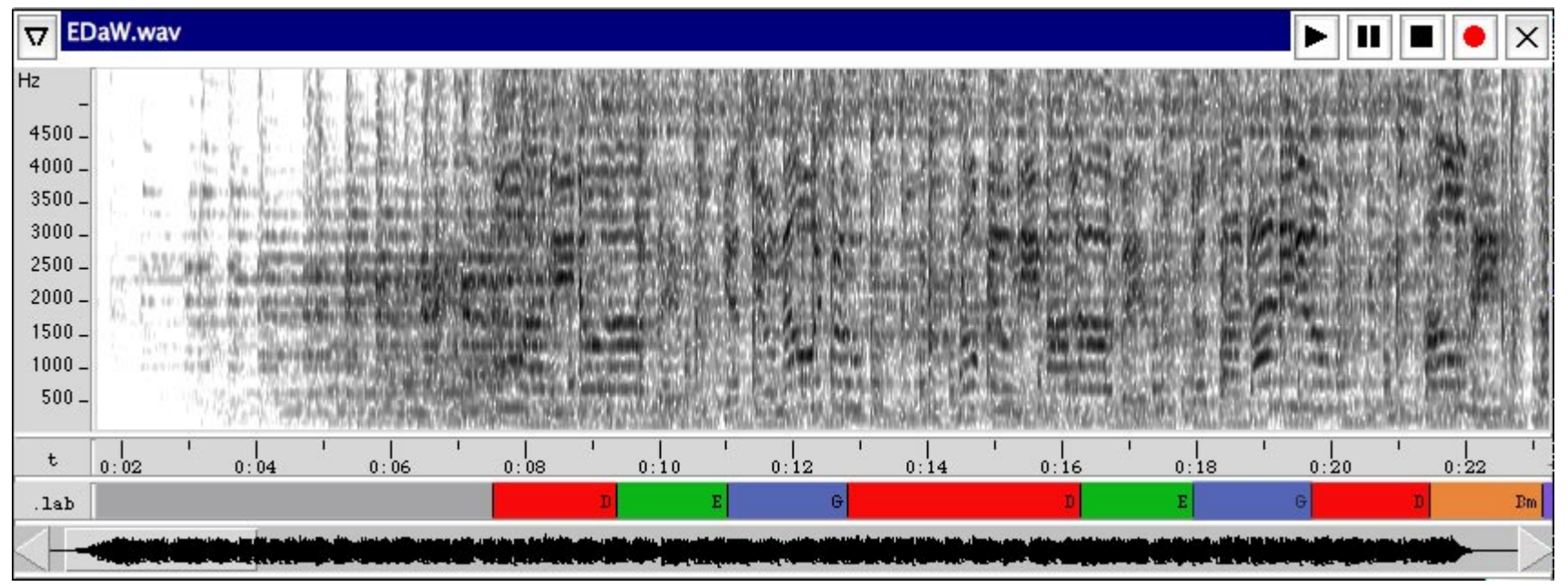

- continuous signal $\rightarrow$ discrete labels

- an essential part of understanding? "information extraction"

- Sound is a challenging domain

- sounds can be highly variable

- human listeners are extremely adept 


\section{Pattern classification}

- Classes are defined as distinct region in some feature space

- e.g. formant frequencies to define vowels

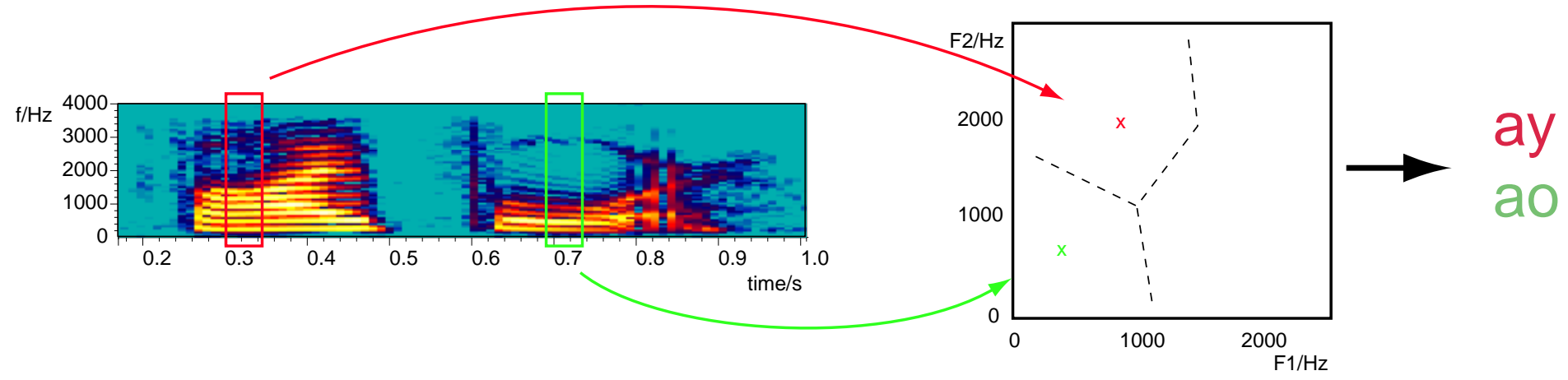

- Issues

- finding segments to classify

- transforming to an appropriate feature space

- defining the class boundaries

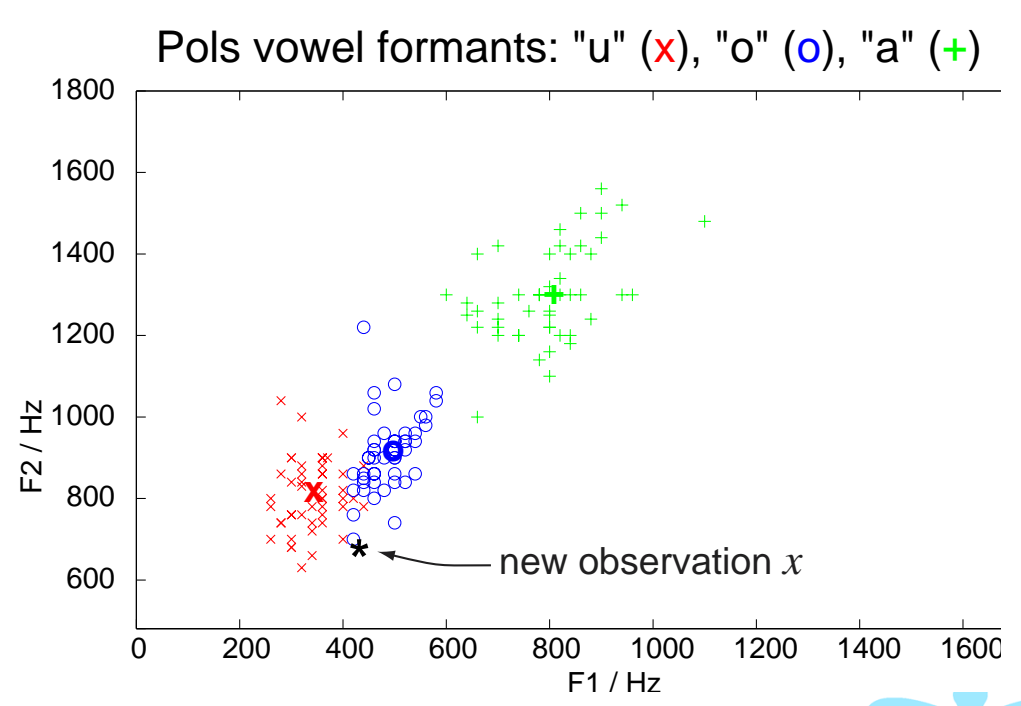




\section{Classification system parts}

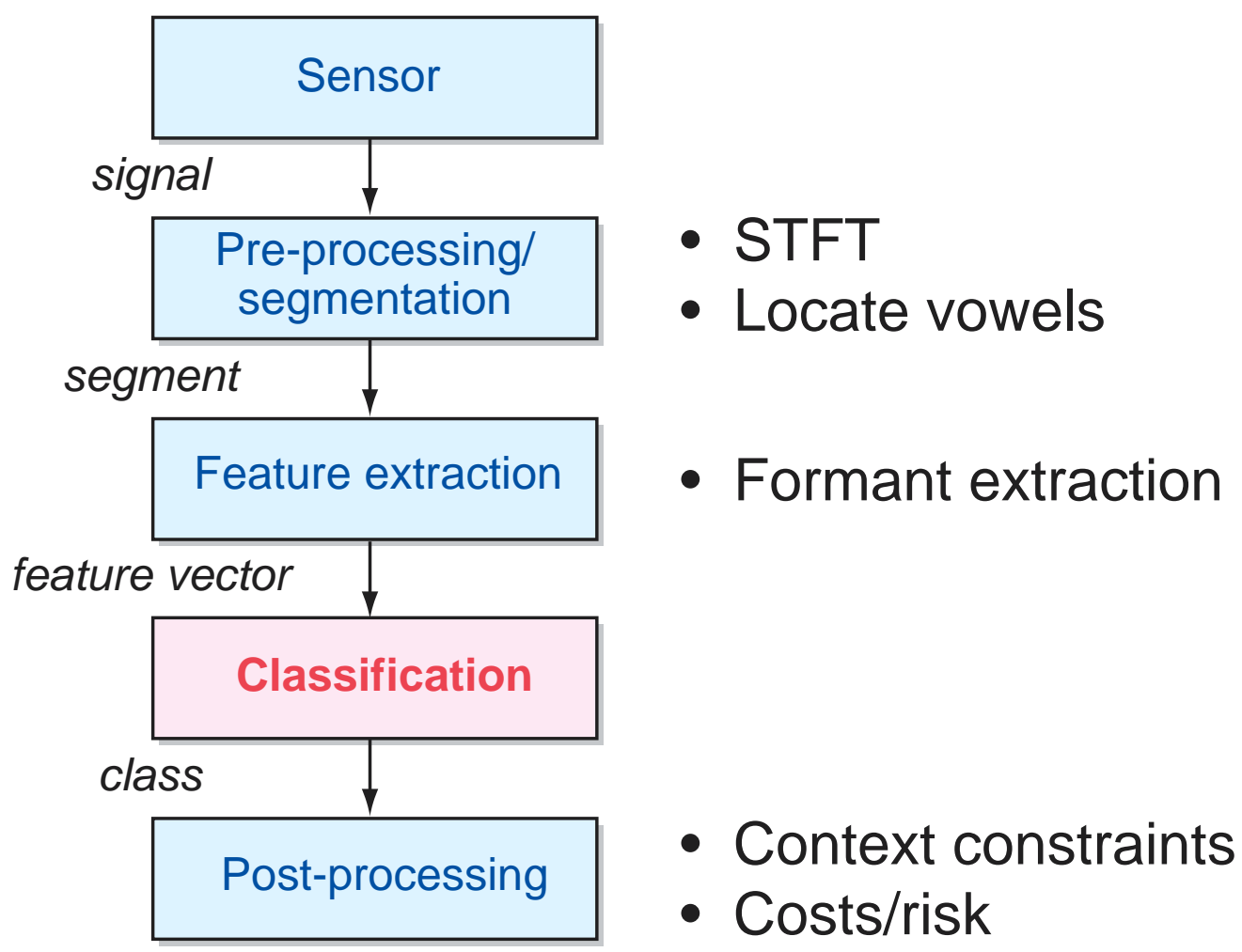




\section{Feature extraction}

- Feature choice is critical to performance

- make important aspects explicit, remove irrelevant details

- 'equivalent' representations can perform very differently in practice

- major opening for domain knowledge ("cleverness")

- Mel-Frequency Cepstral Coefficients (MFCCs): Ubiquitous speech features

- DCT of log spectrum on 'auditory' scale

- approximately decorrelated ...

Mel Spectrogram

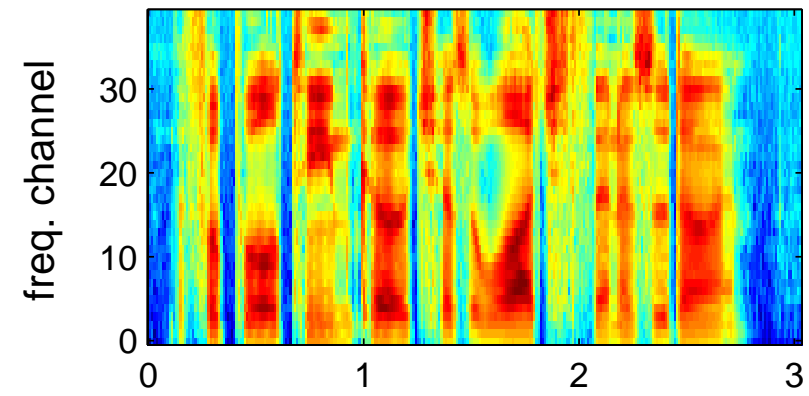

MFCCs

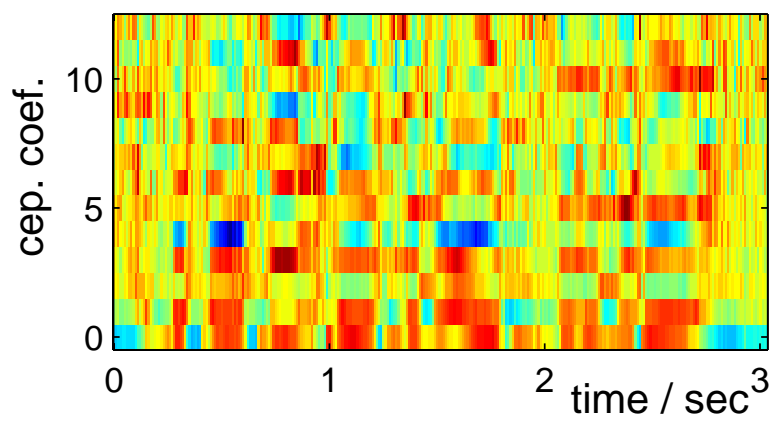




\section{Statistical Interpretation}

- Observations are random variables whose distribution depends on the class:

Class Observation

$\omega_{i}$

$x$

(hidden)

discrete continuous

- Source distributions $p\left(x \mid \omega_{i}\right)$

- reflect variability in feature

- reflect noise in observation

- generally have to be estimated from data (rather than known in advance)

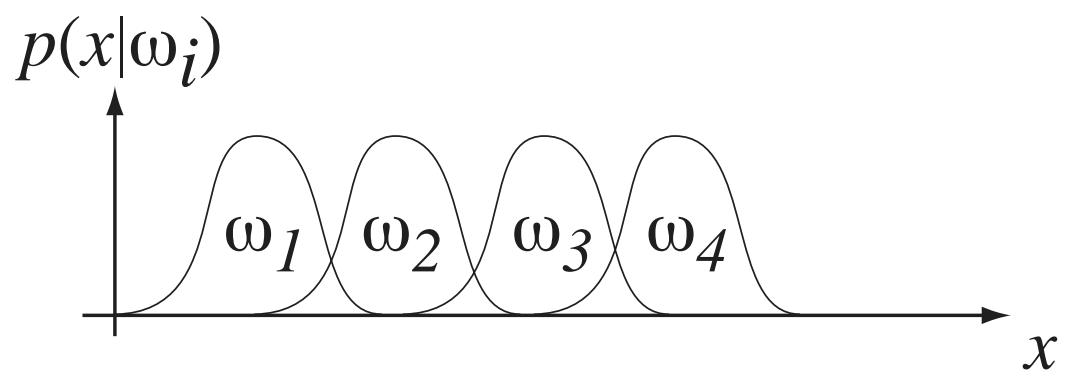




\section{Priors and posteriors}

- Bayesian inference can be interpreted as updating prior beliefs with new information, $x$ :

$$
\underset{\substack{\text { Prior } \\
\text { probability }}}{\operatorname{Pr}\left(\omega_{i}\right)} \cdot \frac{p\left(x \mid \omega_{i}\right)}{\sum_{j} p\left(x \mid \omega_{j}\right) \cdot \operatorname{Pr}\left(\omega_{j}\right)}=\underset{\begin{array}{c}
\text { Lilkelihood } \\
\text { Posteri } \\
\text { 'Evidence' }=\mathrm{p}(\mathrm{x})
\end{array}}{\operatorname{Pr}\left(\omega_{i} \mid x\right)}
$$

- Posterior is prior scaled by likelihood \& normalized by evidence (so $\Sigma$ (posteriors) $=1$ )

- Minimize the probability of error by choosing maximum a posteriori (MAP) class:

$$
\hat{\omega}=\underset{\omega_{i}}{\operatorname{argmax}} \operatorname{Pr}\left(\omega_{i} \mid x\right)
$$




\section{Practical implementation}

- Optimal classifier is $\hat{\omega}=\underset{\omega_{i}}{\operatorname{argmax}} \operatorname{Pr}\left(\omega_{i} \mid x\right)$ but we don't know $\operatorname{Pr}\left(\omega_{i} \mid x\right)$

- So, model conditional distributions $p\left(x \mid \omega_{i}\right)$ then use Bayes' rule to find MAP class

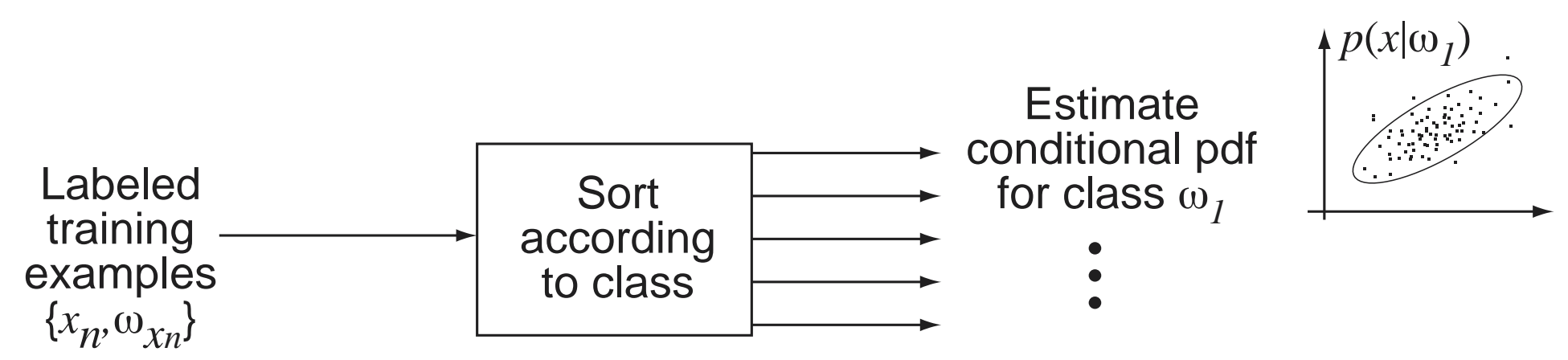




\section{Gaussian models}

- Model data distributions via parametric model

- assume known form, estimate a few parameters

- E.g. Gaussian in 1 dimension:

$$
p\left(x \mid \omega_{i}\right)=\frac{1}{\sqrt{2 \pi} \sigma_{i}} \cdot \exp \left[-\frac{1}{2}\left(\frac{x-\mu_{i}}{\sigma_{i}}\right)^{2}\right]
$$

- For higher dimensions, need mean vector $\mu_{i}$ and $d \times d$ covariance matrix $\Sigma_{i}$
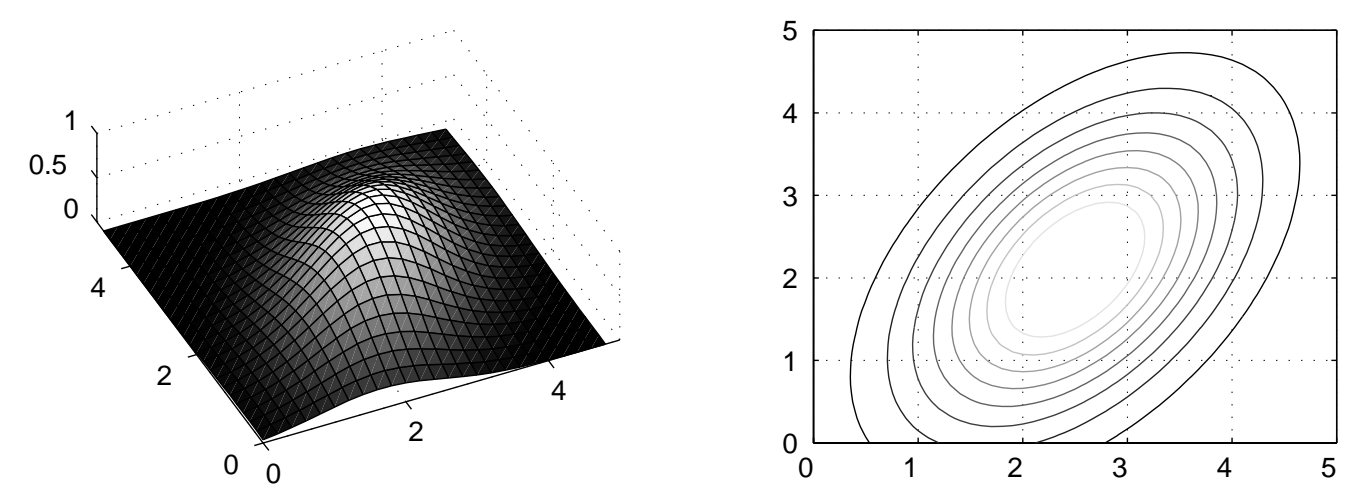

- Fit more complex distributions with mixtures... 


\section{Gaussian models for formant data}
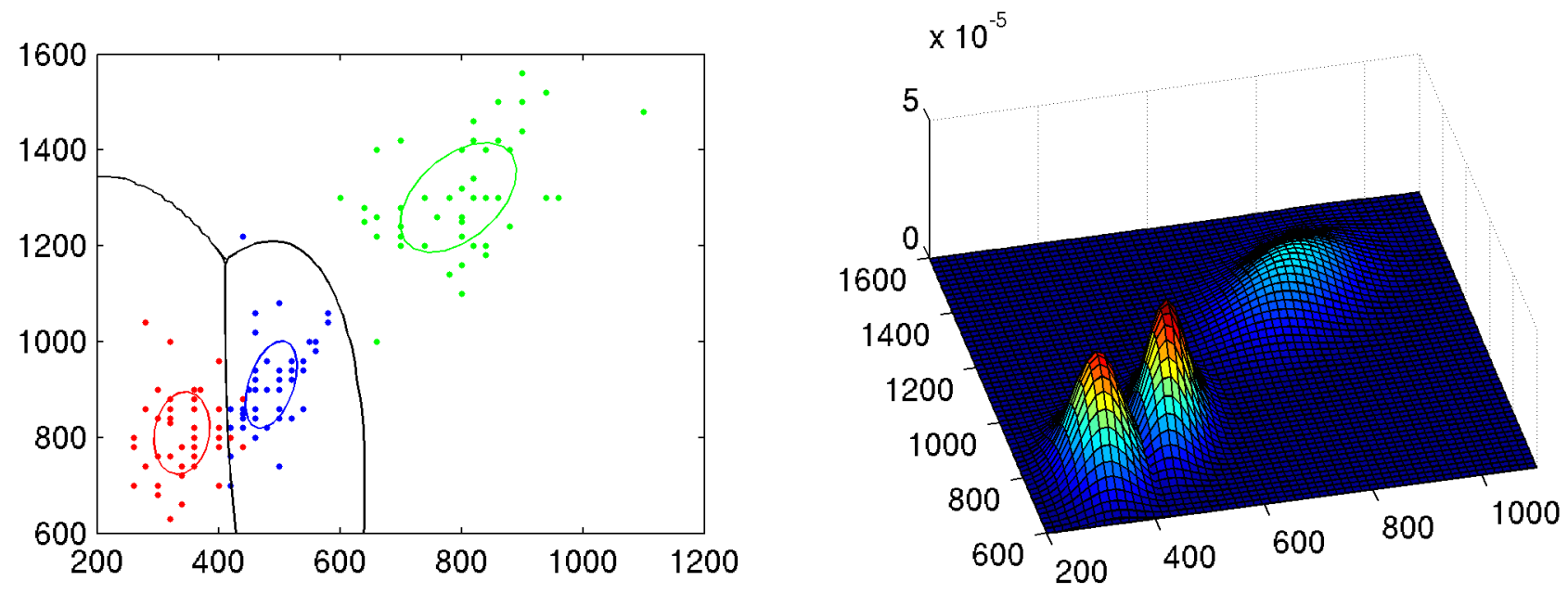

- Single Gaussians a reasonable fit for this data

- Extrapolation of decision boundaries can be surprising 


\section{Outline}

(1) Pattern Recognition for Sounds

2 Speech Recognition

- How it's done

- What works, and what doesn't

(3) Other Audio Applications

(4) Observations and Conclusions 


\section{How to recognize speech?}

- Cross correlate templates?

- waveform?

- spectrogram?

- time-warp problems

- Classify short segments as phones (or ...), handle time-warp later

- model with slices of $~ 10 \mathrm{~ms}$

- pseudo-piecewise-stationary model of words:

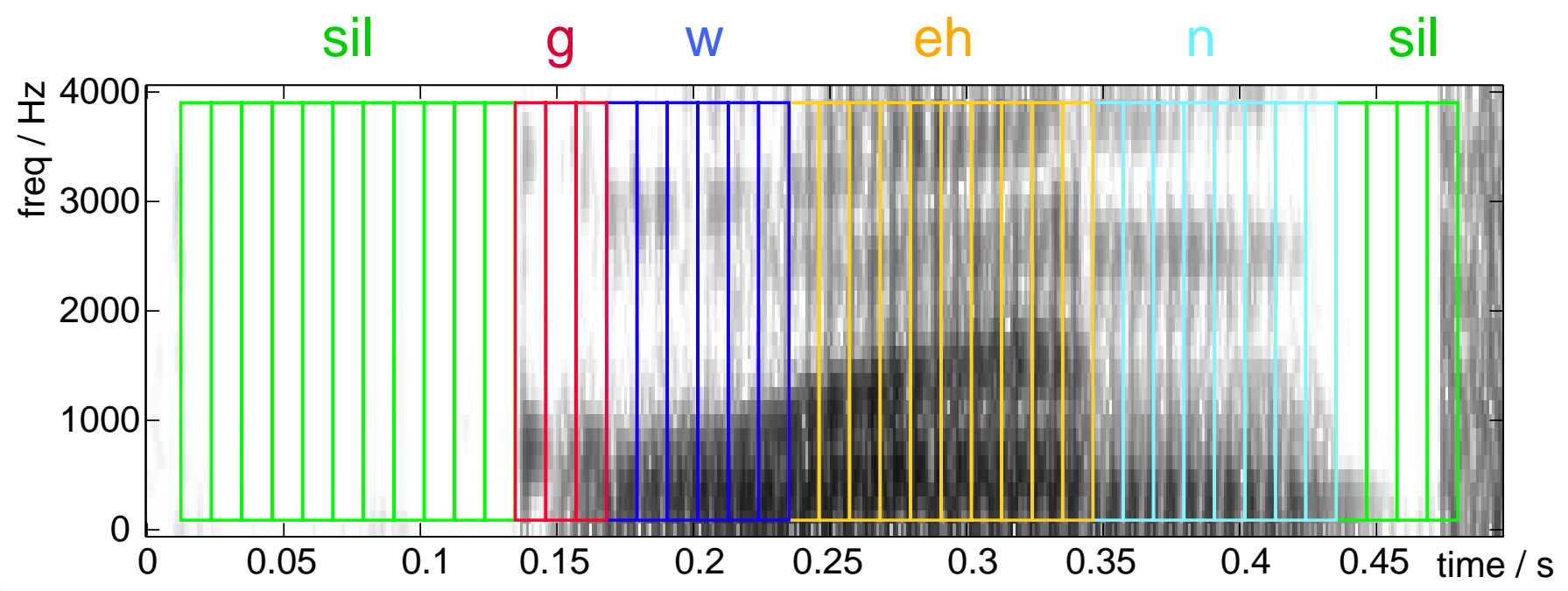




\section{Speech Recognizer Architecture}

- Almost all current systems are the same:

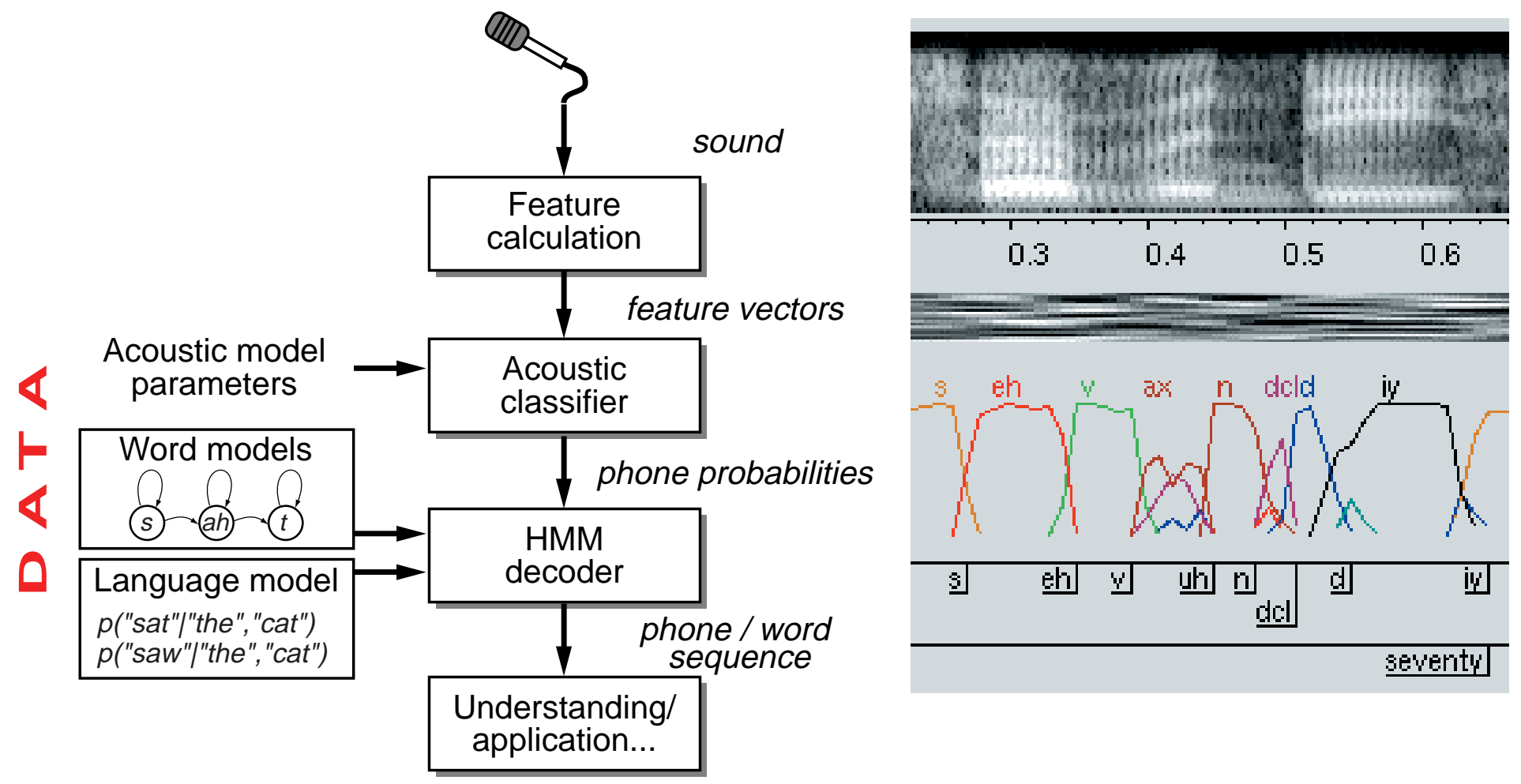

- Biggest source of improvement is increase in training data

- .. along with algorithms to take advantage 


\section{Speech: Progress}

- Annual NIST evaluations

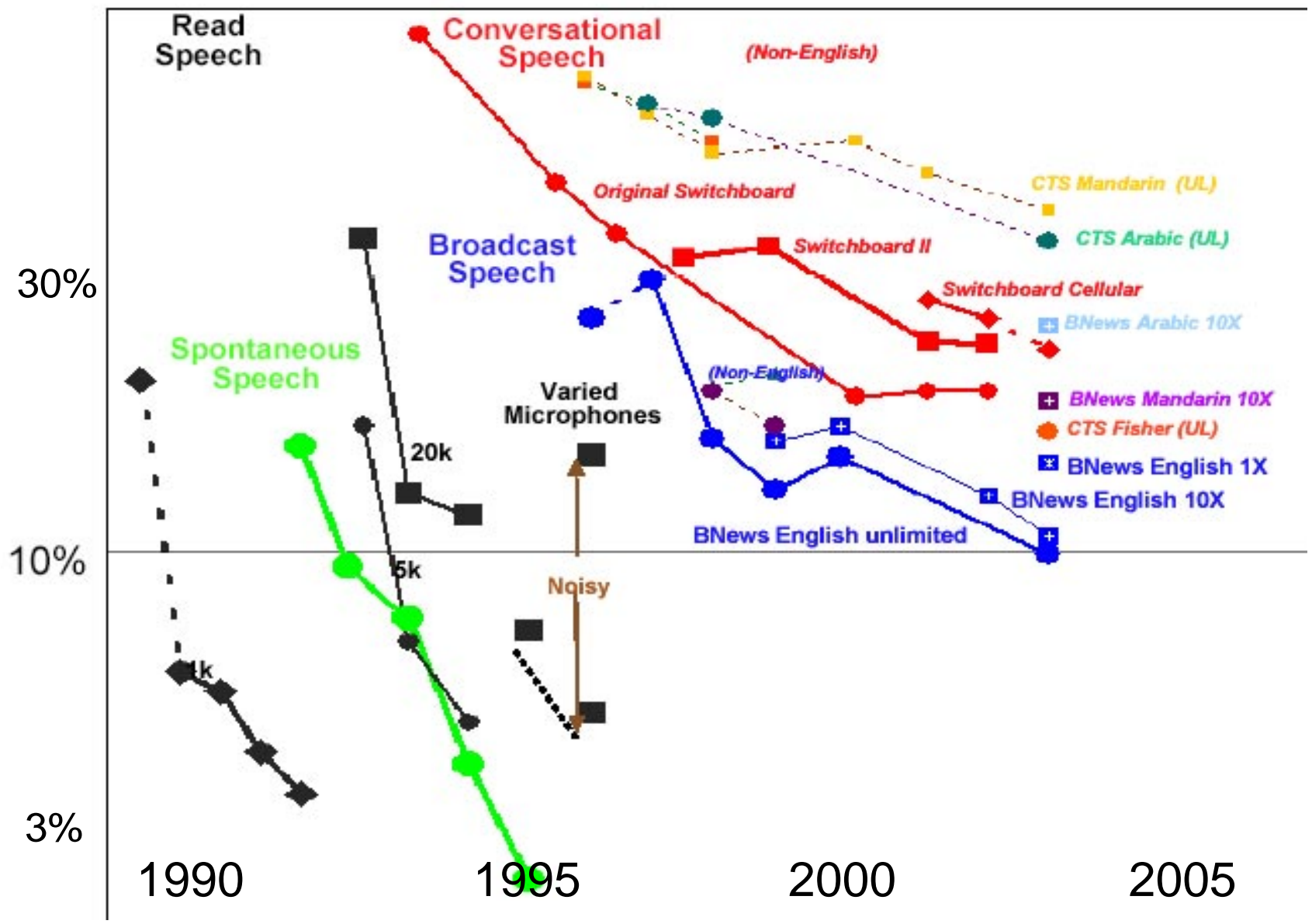

- steady progress (?), but still order(s) of magnitude worse than human listeners 


\section{Speech: Problems}

- Natural, spontaneous speech is weird!

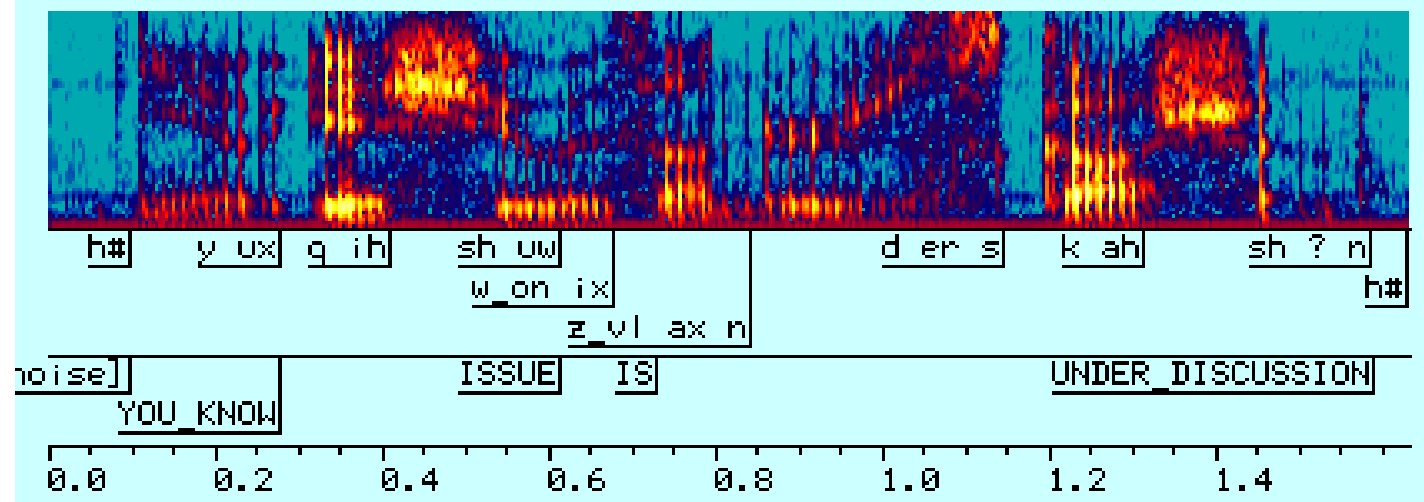

- coarticulation

- deletions

- disfluencies

$\rightarrow$ is word transcription even a sensible approach?

- Other major problems

- speaking style, rate, accent

- environment / background... 


\section{Speech: What works, what doesn't}

- What works: Techniques:

- MFCC features + GMM/HMM systems trained with Baum-Welch (EM)

- Using lots of training data

\section{Domains:}

- Controlled, low noise environments

- Constrained, predictable contexts

- Motivated, co-operative users

- What doesn't work: Techniques:

- rules based on 'insight'

- perceptual representations (except when they do...)

\section{Domains:}

- spontaneous, informal speech

- unusual accents, voice quality, speaking style

- variable, high-noise background / environment 


\section{Outline}

(1) Pattern Recognition for Sounds

(2) Speech Recognition

(3) Other Audio Applications

- Meeting recordings

- Alarm sounds

- Music signal processing

(4) Observations and Conclusions 
Other Audio Applications: ICSI Meeting Recordings corpus

- Real meetings, 16 channel recordings, 80 hrs

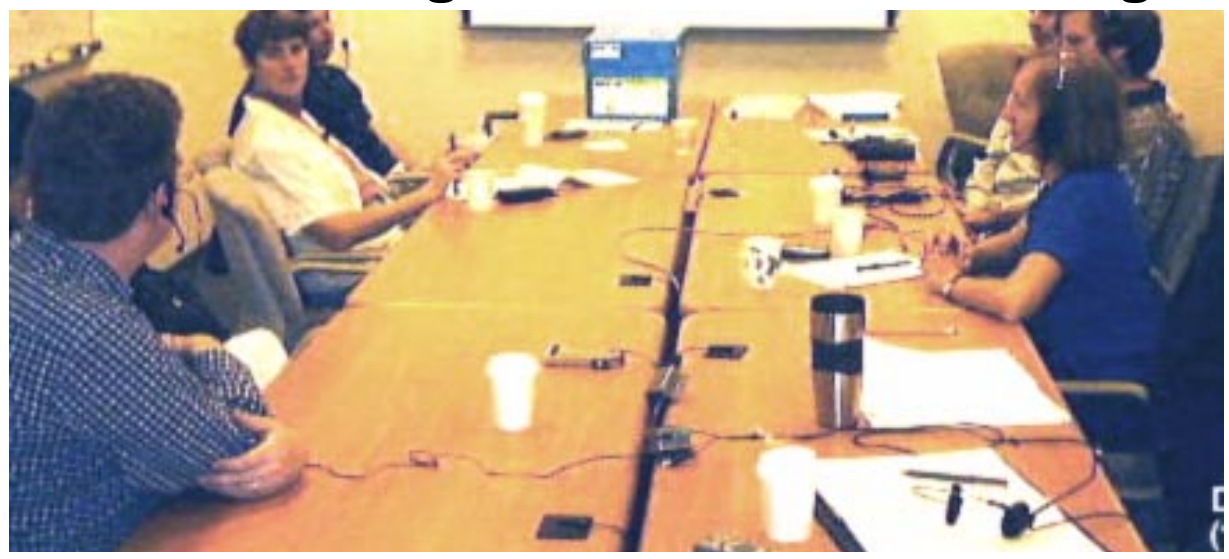

- released through NIST/LDC

- Classification e.g.: Detecting emphasized utterances based on $\mathbf{f}_{0}$ contour (Kennedy \& Ellis '03)

- per-speaker normalized f0 as unidimensional feature $\rightarrow$ simple threshold classification
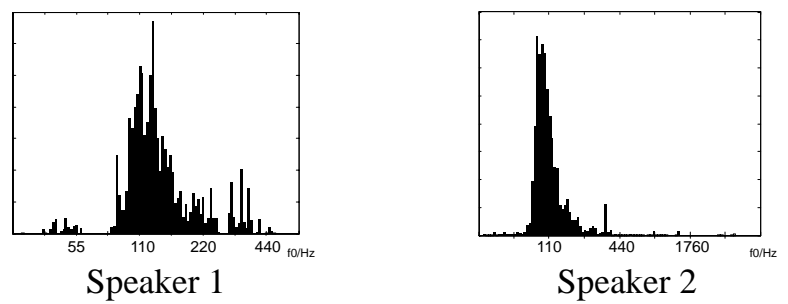


\section{Personal Audio}

- LifeLog / MyLifeBits / Remembrance Agent:

- easy to record everything you hear

- Then what?

- prohibitive to review

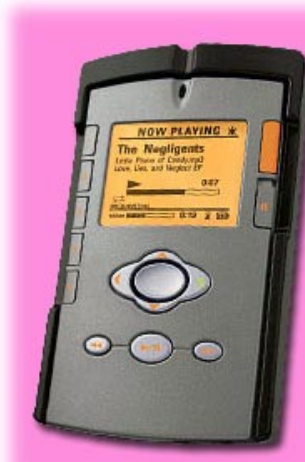

- applications if access easier?

- Automatic content analysis / indexing...
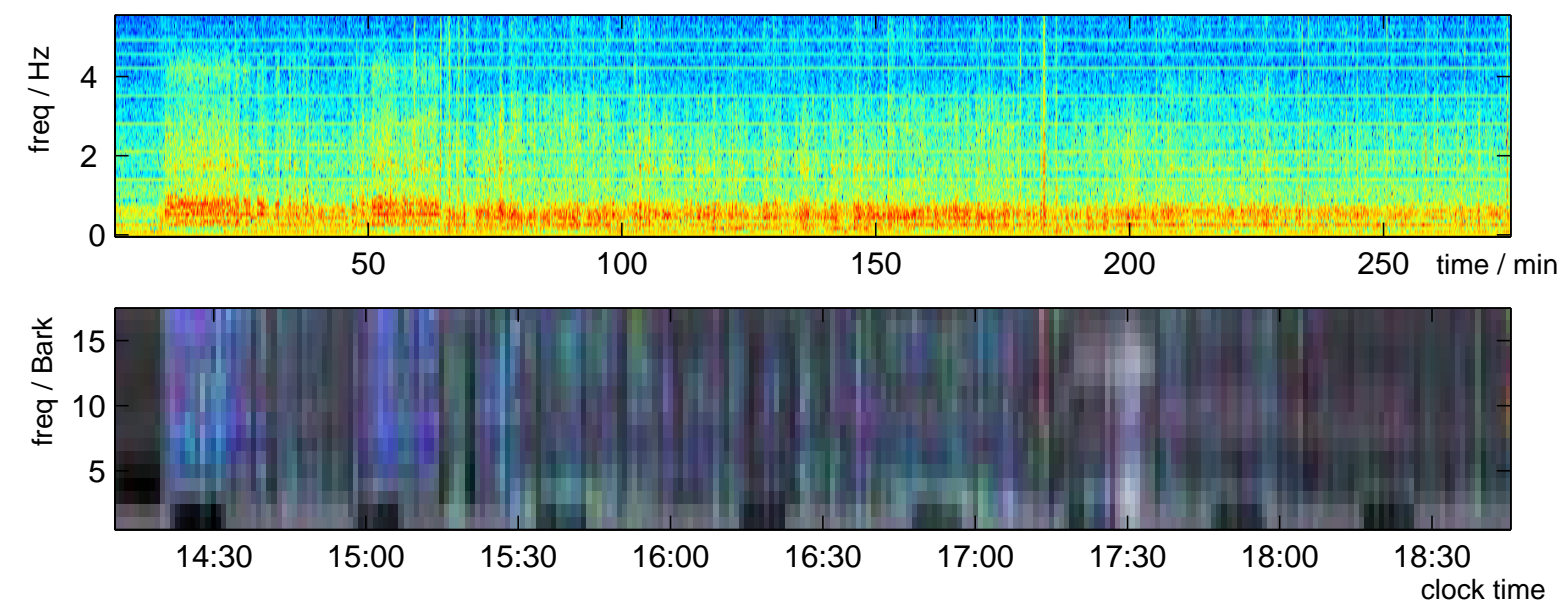

- find features to classify into e.g. locations 


\section{Alarm sound detection}

- Alarm sounds have particular structure

- clear even at low SNRs

- potential applications...

Restaurant+ alarms (snr 0 ns 6 al 8)

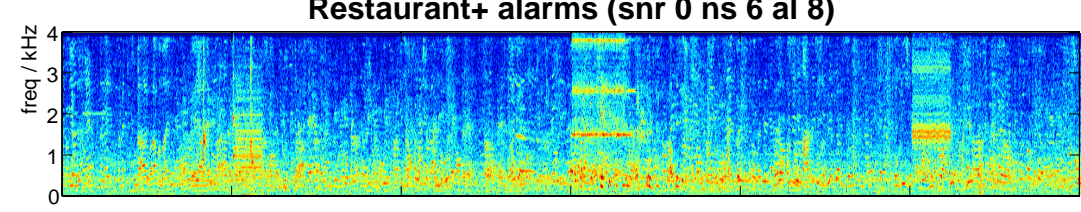

- Contrast two systems: (Ellis '01)

- standard, global features, $P(X \mid M)$

- sinusoidal model, fragments, $P(M, S \mid Y)$

MLP classifier output

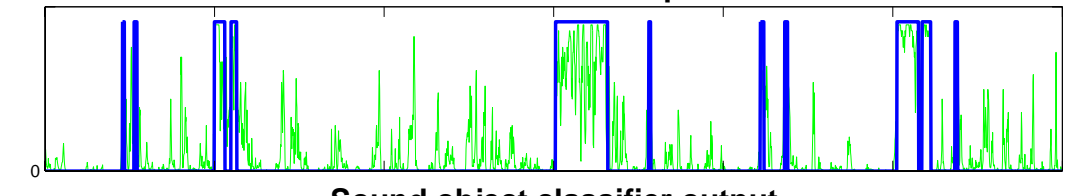

Sound object classifier output

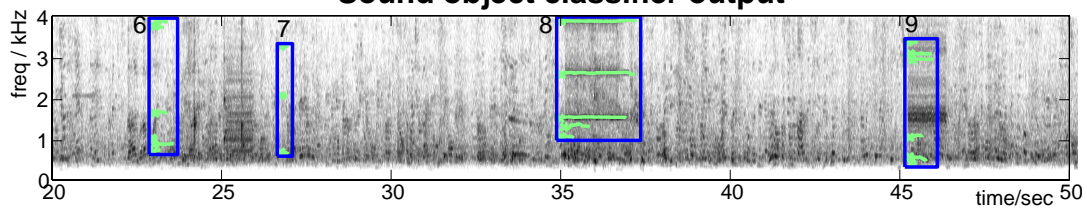

- error rates high, but interesting comparisons... 


\section{Music signal modeling}

- Use "machine listener" to navigate large music collections

- e.g. unsigned bands on MP3.com

- Classification to label:

- notes, chords, singing, instruments

- .. information to help cluster music

- "Artist models" based on feature distributions
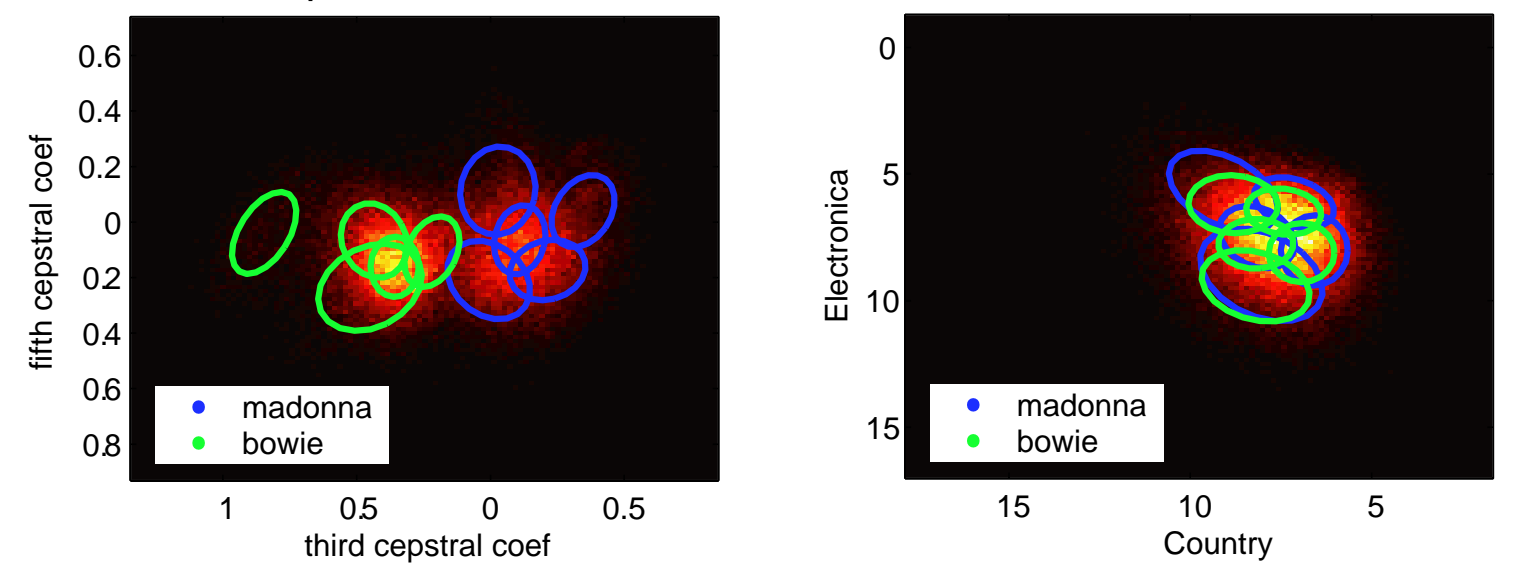

- measure similarity between users' collections and new music? (Berenzweig \& Ellis '03) 


\section{Outline}

(1) Pattern Recognition for Sounds

(2) Speech Recognition

(3) Other Audio Applications

(4) Observations and Conclusions

- Model complexity

- Sound mixtures 
Observations and Conclusions: Training and test data

- Balance model/data size to avoid overfitting:

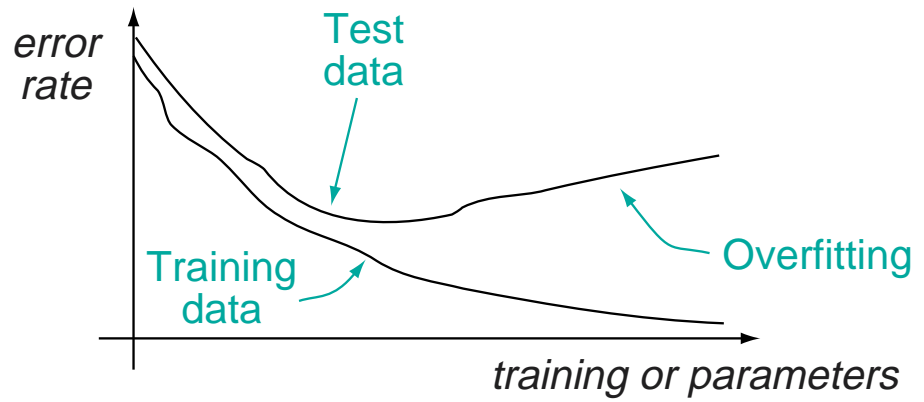

- Diminishing returns from more data:

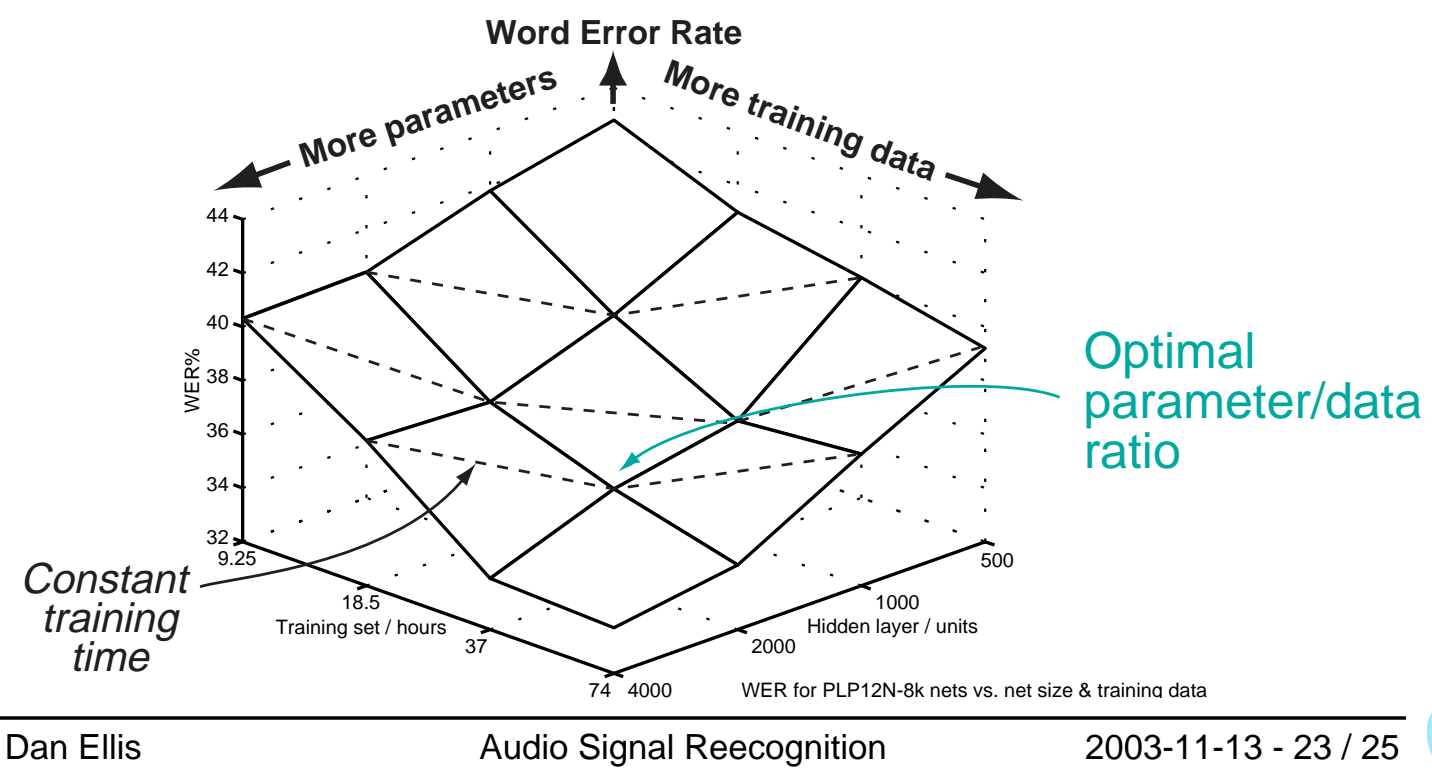




\section{Beyond classification}

- "No free lunch":

\section{Classifier can only do so much}

- always need to consider other parts of system

- Features

- impose ceiling on system performance

- improved features allow simpler classifiers

- Segmentation / mixtures

- e.g. speech-in-noise: only subset of feature dimensions available $\rightarrow$ missing-data approaches...

\section{$Y(t)$}
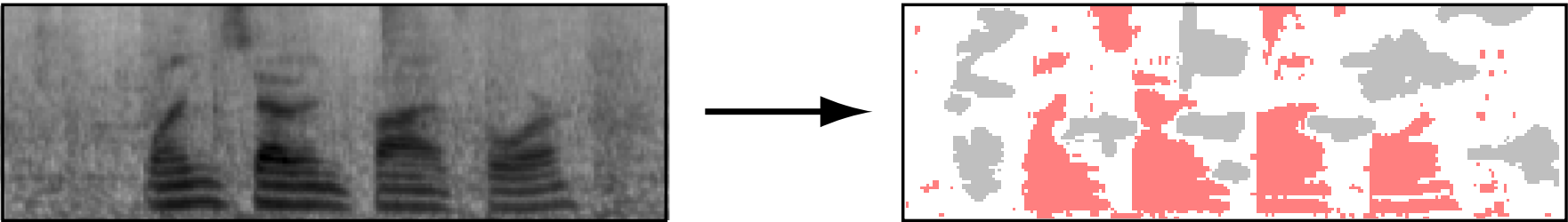

$S_{2}(t)$

$S_{7}(t)$ 


\section{Summary}

- Statistical Pattern Recognition

- exploit training data for probabilistically-correct classifications

- Speech recognition

- successful application of statistical PR

- .. but many remaining frontiers

- Other audio applications

- meetings, alarms, music

- classification is information extraction

- Current challenges

- variability in speech

- acoustic mixtures 


\section{Extra slides}




\section{Neural network classifiers}

- Instead of estimating $p\left(x \mid \omega_{i}\right)$ and using Bayes, can also try to estimate posteriors $\operatorname{Pr}\left(\omega_{i} \mid x\right)$ directly (the decision boundaries)

- Sums over nonlinear functions of sums give a large range of decision surfaces...

- e.g. Multi-layer perceptron (MLP):

$y_{k}=F\left[\sum_{j} w_{j k} \cdot F\left[\sum_{j} w_{i j} x_{i}\right]\right]$

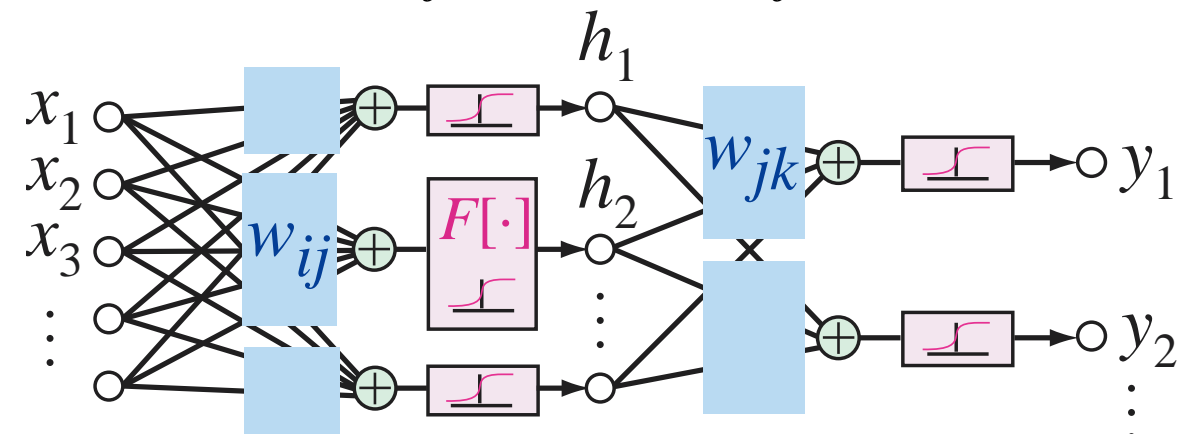

Input

layer

Hidden

layer
Output

layer

- Problem is finding the weights $w_{i j} \ldots$ (training) 


\section{Neural net classifier}
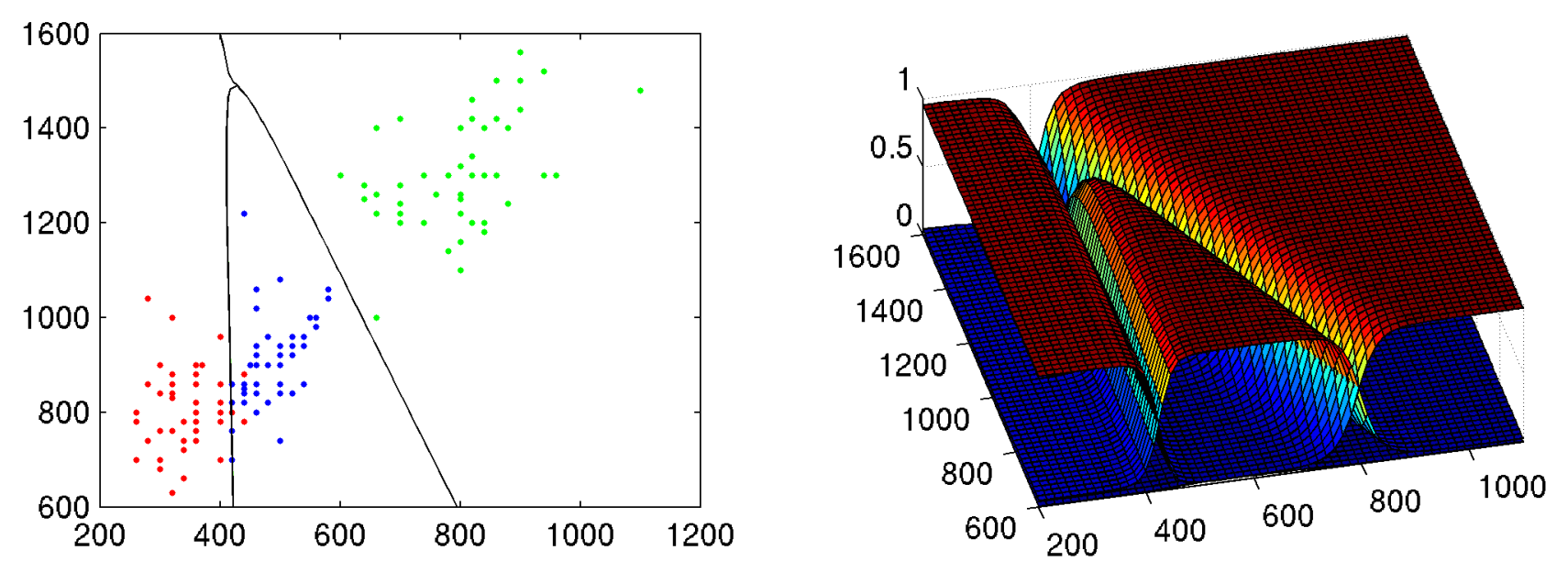

- Models boundaries, not density $p\left(x \mid \omega_{i}\right)$

- Discriminant training

- concentrate on boundary regions

- needs to see all classes at once 


\section{Why is Speech Recognition hard?}

- Why not match against a set of waveforms?

- waveforms are never (nearly!) the same twice

- speakers minimize information/effort in speech

- Speech variability comes from many sources:

- speaker-dependent (SD) recognizers must handle within-speaker variability

- speaker-independent (SI) recognizers must also deal with variation between speakers

- all recognizers are afflicted by background noise, variable channels

$\rightarrow$ Need recognition models that:

- generalize i.e. accept variations in a range, and

- adapt i.e. 'tune in' to a particular variant 


\section{Within-speaker variability}

- Timing variation:

- word duration varies enormously

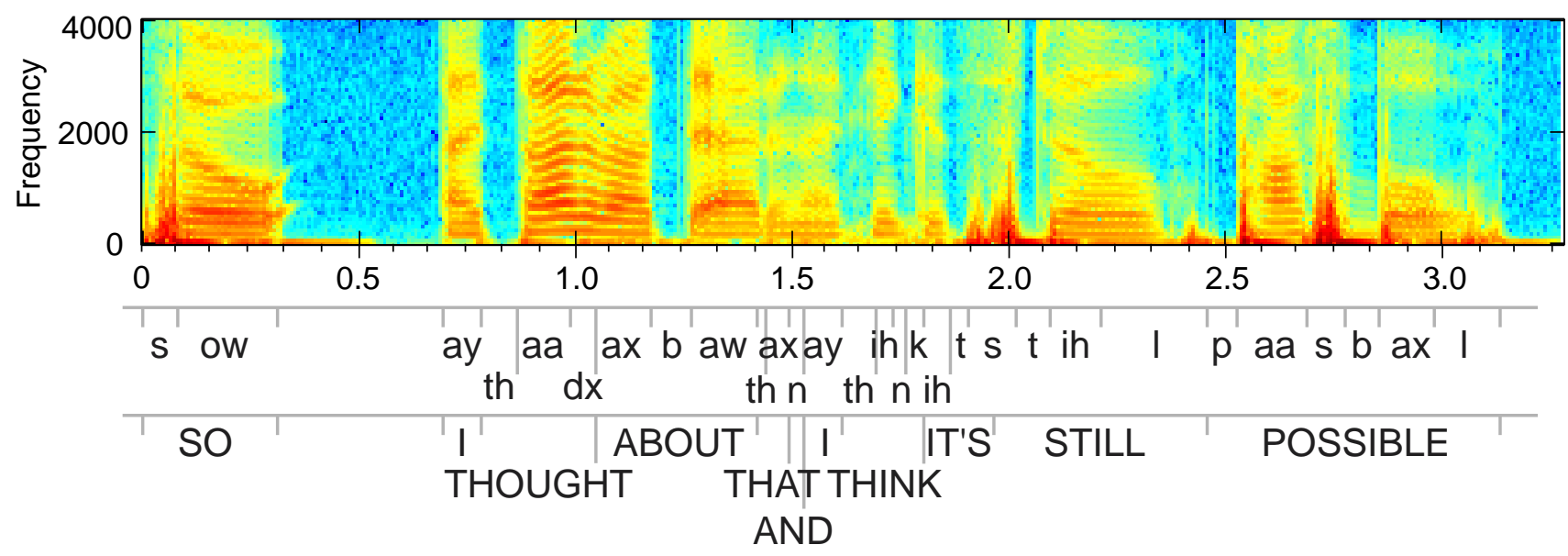

- fast speech 'reduces' vowels

- Speaking style variation:

- careful/casual articulation

- soft/loud speech

- Contextual effects:

- speech sounds vary with context, role: "How do you do?" 


\section{Between-speaker variability}

- Accent variation

- regional / mother tongue

- Voice quality variation

- gender, age, huskiness, nasality

- Individual characteristics

- mannerisms, speed, prosody

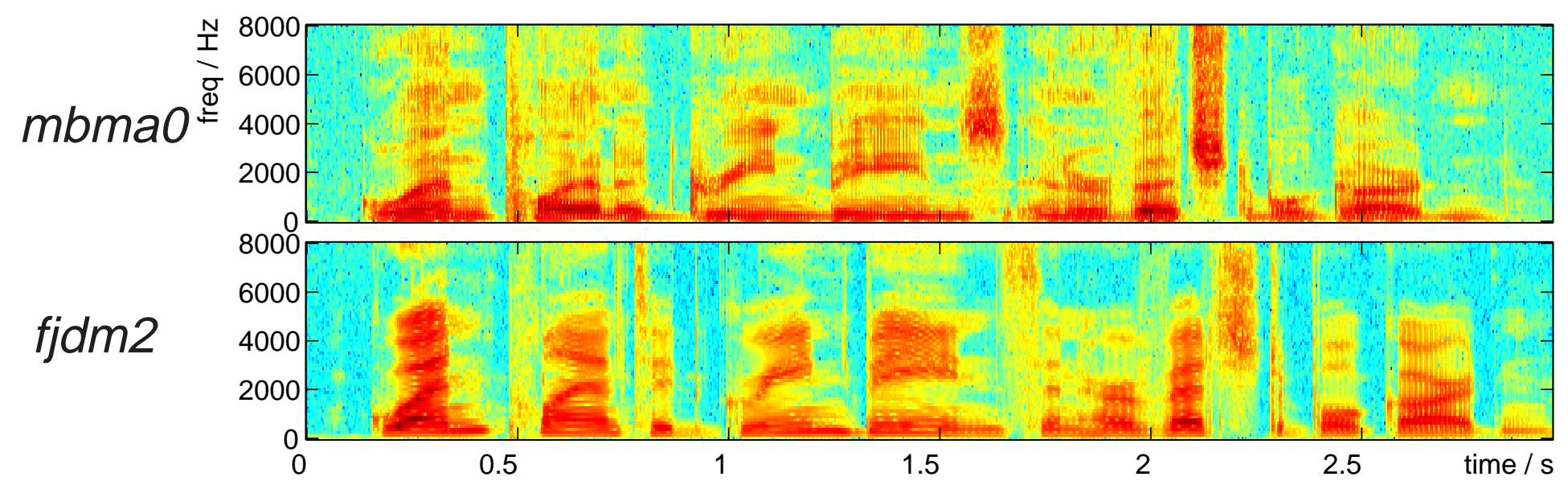




\section{Environment variability}

- Background noise

- fans, cars, doors, papers

- Reverberation

- 'boxiness' in recordings

- Microphone channel

- huge effect on relative spectral gain
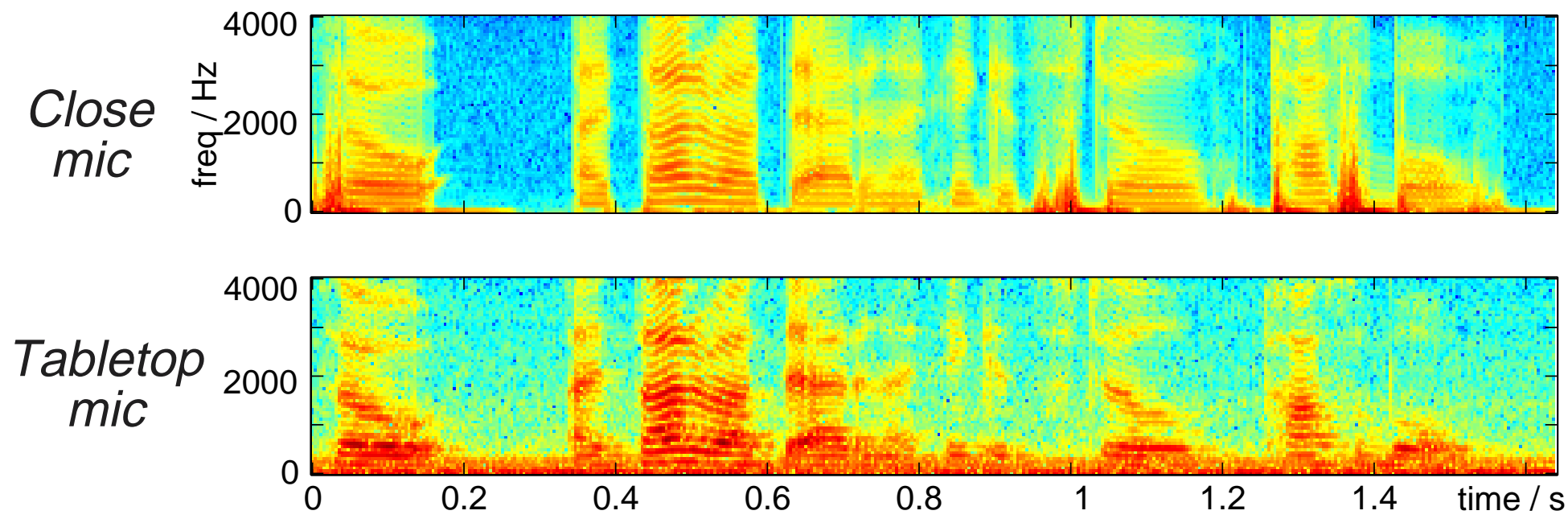